\title{
Dynamic Tangible User Interface Palettes
}

\author{
Martin Spindler ${ }^{1}$, Victor Cheung ${ }^{2}$, and Raimund Dachselt ${ }^{3}$ \\ ${ }^{1}$ User Interface \& Software Engineering Group, University of Magdeburg, Germany \\ ${ }^{2}$ Collaborative Systems Laboratory, University of Waterloo, Canada \\ ${ }^{3}$ Interactive Media Lab Dresden, Technische Universität Dresden, Germany \\ spindler@ovgu.de, victor.cheung@uwaterloo.ca, dachselt@acm.org
}

\begin{abstract}
Graphics editors often suffer from a large number of tool palettes that compete with valuable document space. To address this problem and to bring back physical affordances similar to a painter's palette, we propose to augment a digital tabletop with spatially tracked handheld displays. These displays are dynamically updated depending on their spatial location. We introduce the concept of spatial Work Zones that take up distinct 3D regions above the table surface and serve as physical containers for digital content that is organized as stacks of horizontal layers. Spatial Work Zones are represented either by physical objects or on-screen on the tabletop. Associated layers can be explored fluently by entering a spatial Work Zone with a handheld display. This provides quick access and seamless changes between tools and parts of the document that are instantly functional, i.e., ready to be used by a digital pen. We discuss several use cases illustrating our techniques and setting them into context with previous systems. Early user feedback indicates that combining dynamic GUI functionality with the physicality of spatially tracked handheld displays is promising and can be generalized beyond graphics editing.
\end{abstract}

Keywords: Tangible user interface palettes, spatial Work Zones, tabletop displays, dynamic pen heads, spatial management.

\section{Introduction}

Graphical User Interfaces (GUI) have been commonly used as the medium of interaction with computer applications due to their iconic appearance and ease of control. With GUIs, tools are often organized as tool palettes that group similar functionality within separate windows floating on top of the document. One drawback, however, is that these palettes take up the very same display space as the document does. This can be a major issue when applications support a large number of different tools. A typical example for this are graphics editors, such as Adobe Photoshop and Illustrator. Due to the wide spectrum of functionality - e.g., navigation, layer handling, system control, selection management, brush and color selection, etc. - many tool palettes are often displayed simultaneously, cluttering valuable display space. A common way to approach this problem is to rely on more transient interfaces, such as context menus, menus from the menu bar, or see-through widgets [5] that do not permanently occlude 
objects of interest. Unfortunately, this still forces users to spend considerable time and effort on organizing and navigating through tool hierarchies, thus taking away mental resources from their actual goals. One naïve solution to this problem is to provide more room for the interface and document by using larger displays, such as digital tabletops. Yet, the underlying conflict still remains: the strife between user interface and document over the very same display space.

Meanwhile, recent developments show that large interactive displays are gaining popularity in a variety of application domains. This is due to the fact that they fuse input and output in the same space, thus allowing for a more natural and intuitive interaction by using the concepts of direct pointing and manipulation. This is also supported by the ample size of such displays that provides an adequate environment for co-located collaborations. Previously, several projects have focused on the design and implementation of such displays in different configurations, either horizontally (e.g., interactive tables [8]), vertically (e.g., digital whiteboards [9]), or tilted (e.g., sketching table [25]). Others have created mixed display environments that benefit users with the best of each individual setup, for instance, by using display walls for presentation tasks, tabletops for collaborative work on shared documents, and smaller mobile displays for personal tasks [19, 20].

Apparently, content transfer between devices plays a vital role in such mixed display environments. For that, a variety of techniques have been presented aiming at an almost ubiquitous handling of content between the "digital" and the "analog" world, e.g., by using paper-like digital interfaces $[13,15]$. Others, in turn, have demonstrated how the spatial position and orientation of such paper-like displays can directly be utilized for interaction purposes - used, for example, for zooming in/out details of large image documents that are displayed on a tabletop by moving a handheld digital paper screen up and down [29]. Most of these projects have focused on the problem of how to interact with and move data between different workspaces. Yet there is little work on investigating how spatial movements and arrangements of tools facilitate the work practice, which is the goal of our work.

To address this problem, we build on the benefits of tangible displays in a tabletop environment that extend the interactive space to dynamically updated and spatially tracked handheld displays. Our contributions are: (1) the physical separation of document and tools by using digital pen-enabled paper-like handheld displays, (2) the use of the 3D space above the table for interaction by introducing spatial Work Zones that provide quick and easy access to either tools or the document as well as fluid switches between the two of them, and (3) the demonstration of their usefulness by several use cases and a prototypic implementation.

In the remainder of the paper, we first review related work and identify a gap between the "analog" and "digital" world by using the example of graphics editing. Next, we propose our concept of Tangible User Interface Palettes (TUIP) that aims at closing this gap. This includes the description of the design space and our concept of spatial Work Zones. We then present several use cases that demonstrate how TUIP principles can help users organize and work with complex graphical user interfaces, as illustrated with a graphics editor application. After a brief walk through the technical setup, we report early user feedback and finish with some concluding remarks. 


\section{Related Work}

Our work is based on a variety of previous research that we extend and combine within a single system. This includes conceptual as well as technical aspects.

\subsection{Tangible User Interfaces: Physical Separation of Tool and Document Space}

Merging the digital world with the physical world is the vision of ubiquitous computing, as defined by Weiser [35]. This concept was adapted by Ishii's and Ullmer's tangible user interfaces (TUIs) [17], where interaction with digital information is provided through physical manipulation of real-world objects (tangible objects). TUIs have been used to cope with the conflict between document and tools over the same display space by supplying additional multi-purpose tangible objects that users can manipulate with their hands, but without involving the document view. One example for this is using real brushes in different sizes and shapes, such as demonstrated with IntuPaint [34], though this still relies on virtual color widgets on the main screen. By adding interactive components to a digital pen, such as multi-touch capability to a pen's barrel, as demonstrated by Song et al. [28], users can switch between different interaction modes or drawing styles. I/O brush [27] goes a step further and lets children draw with digital colors and textures that they pick up from the real world.

Tangible GUIs aim at bridging the familiarity of conventional GUIs and the rich affordances of tangible objects. For example, controls can easily be organized and passed around. In addition, the main display is less cluttered, as most of the GUI components can be "detached" from the main display. In Phidgets [10], Greenberg and Fitchett demonstrated the support for user-adaptation of physical UI components using wired controls. The VoodooSketch project by Block et al. [6] extended a tabletop environment with printed paper-based GUI palettes that were physically separated from the main display, and supported live sketching of custom-shaped controls and their configuration by handwritten labels or prefabricated components (e.g., buttons and sliders) on physical palettes. However, once created, these interface palettes remained static until they were physically altered or thrown away.

In contrast, our work is based on digital paper-like displays, where digital content is dynamically projected onto physical palettes, and thus can easily be adapted and manipulated with immediate visual feedback. In this way, our project shares principles from the Paper Windows project by Holman et at. [15] that addresses the capturing of physical affordances of paper in a digital world.

\subsection{Projective Display Technology}

Compared with active displays, e.g., smart phones and tablets, projective (paper-like) displays are typically more lightweight, customizable, almost "tech-free", and are inexpensive to reproduce once the system is running with at least one display. Most projective display systems follow the same technical approach. First, the spatial positions of one or more handheld projection screens are determined, e.g., optically by using infrared (IR) cameras and IR-reflective markers [15]. Then content is projected 
onto the displays by a stationary projector that either resides above or in front of the workspace (e.g. ceiling-mounted [15, 24, 29]) or even below a tabletop surface, such as demonstrated in Second Light [18] and UlteriorScape [20]. One of the great potentials of projective displays is its support for digital pen and paper technology (e.g., Anoto-based [1]), which further helps merge "analog" with "digital" content, making it an ideal technical base for ubiquitous computing. On the technical side, our TUIP prototype utilizes projective paper-like display technology, in particular the one used and presented in our own previous work PaperLens [29].

\subsection{Tangible Magic Lens Interaction (in a Tabletop Environment)}

Inspired by the notion of see-through interfaces [5], tangible user interfaces can also be spatially tracked handheld displays (Tangible Displays) that serve as Tangible Magic Lenses into a virtual world. Tangible displays have successfully been used in tabletop environments, for example, in the metaDESK [33] project, where users can explore a virtual 3D campus by moving an arm-mounted TFT-display above a tabletop showing a digital 2D map of the campus. In PaperLens [29], we demonstrated how such Tangible Magic Lenses (handheld displays) can be used to navigate through individual parts of a large image document that is displayed on the tabletop. Here, lifting and lowering a handheld display correlates with instant zooming, whereas moving a display horizontally results in panning. In PaperLens, all spatial interaction was implicit, meaning that any positional change of a display triggered immediate updates on its screen. With Tangible Views [30], we introduced the concept of "freezing" that allowed for actively locking the screen content by holding a button, thus preventing any visual updates as long as the button was pushed. Yet, implicit spatial input based interaction still remained the default. For TUIP, we use similar techniques for document navigation. However, to better meet the requirements of graphics editor applications, where unintended navigation can be very disturbing, users must explicitly activate any spatially based navigation by holding and pushing a physical button attached to a handheld display.

\subsection{Proxemics Interactions}

The field of proxemics interactions originates from studies of interpersonal behavior in relation to physical distances. It has been adapted and widely studied in humancomputer interaction as a means of providing appropriate responses based on user's proximity $[3,14]$. Another adaptation is to make use of the spatial relation between tangibles and adjust their behavior. In their initial work of the Smart-Its project, Holmquist et al. [16] proposed context proximity which connects artifacts based on physical distances and user actions (e.g. shaking the artifacts). Kray et al. [22] investigated the use of spatial regions around mobile devices (tracked using on-screen markers) for content sharing, and demonstrated their benefits on group coordination and social processes. In our work, we combine proxemics interaction principles with tangible display interaction by introducing spatial Work Zones, which are distinct spatial regions above the tabletop with a certain meaning associated to them. 


\subsection{Multi-layer Stack Interaction with Tangible Magic Lenses}

One particular spatial interaction style for tangible displays is multi-layer interaction, such as was proposed and demonstrated by us with PaperLens [29]. The principle idea behind multi-layer interaction is to arrange virtual 2D layers in a vertical stack within the physical 3D space, with each layer consuming a distinct height of the volume. Such multi-layer stacks can be explored by moving handheld displays through it. Vertical movements of a display along the Z-axis allow for selecting a layer, while holding a display at a certain height allows for viewing or working with a particular layer. At the same time, moving the display horizontally allows for exploring a specific layer. In [31], we conducted an extensive user study and derived specific design guidelines for this style of interaction. Later, in LightSpace [36], Wilson and Benko projected spatial vertical menus directly onto the hand of users, thus suggesting a similar way of interaction by revealing menu options when the hand is being moved up and down. For TUIP, we use multi-layer stack interaction principles for organizing related tool palettes or documents within spatial Work Zones.

\section{Identifying the Gaps between Two Opposite Worlds}

We start our exploration by comparing the benefits and weaknesses of tool handling in two opposite worlds: the real (analog) world and the digital world. By using the example of graphics editing, we will identify significant gaps between both worlds. Narrowing these gaps was a major motivation for working on TUIP.

\subsection{Graphics Editing in the Analog World}

Prior to the digital age, painters and graphics artists used to work with real-world tools and painting mediums (e.g., brushes, color palettes and canvases), which were physically separated from each other and could be spatially arranged, grouped, manipulated, and combined within the physical 3D world in a very natural and personalized way. This style of interaction implicitly leverages the whole spectrum of spatial arrangement, which has proven to simplify choice, perception, and even internal computation [21]. After all, in having a body we are spatially located beings. We must always face some direction and therefore have only certain objects in view. Thus, managing the spatial arrangement of items around us is not circumstantial - it is an integral part of the way we think, plan and work. In this spirit, artists freely setup and configure their working environment to fit the task at hand, e.g., by defining and using specific work zones on the desk. This gives them not only fast access to all necessary tools, but also requires them to memorize less - by actively increasing their understanding of the spatial organization in the outside world. This is supported by human perception that can handle a high amount of information in the periphery, even if the focus is on a particular detail [32].

As a downside, working with the analog world is often limited by physical constraints of tools and materials. For example, existing drawings are hard to reproduce and supplies will run out or deteriorate after prolonged use. 


\subsection{Graphics Editing in the Digital World}

In the digital world, graphics artists can reuse, share, modify, and combine digital content very efficiently by using digital tools that simulate analog techniques (e.g. airbrushes, paint brushes, erasers). This increases productivity and even complements the toolbox with novel styles, techniques and filters that were not available before in the analog world. Beyond that, many physical constraints are removed in the digital world. For example, colors never run out and the drawing space is virtually limitless. This is reflected by the extensive set of tools offered by common desktop graphics editors like Adobe Photoshop, Adobe Illustrator and GIMP.

One major disadvantage, however, is the restriction to a two-dimensional (2D) workspace: the digital screen that provides only a spatially constrained peephole into an otherwise large and complex world. This effectively takes away the advantages of spatial arrangement that users are familiar with from the analog world. Two challenges go hand in hand with this: tool management and document navigation.

Challenge 1: Tool Management. The immense amount of digital functionality increases the complexity of tool usage. This results in valuable screen space being cluttered by tools, leaving less space for the actual document. Common approaches to soften (but not solving) this problem include keyboard shortcuts, tool presets, context menus (e.g., Pie/Marking Menus [23]), and menus from the menu bar, i.e., transient interfaces that do not permanently occlude objects of interest - thus saving valuable screen space. Some of these techniques were adapted to multi-touch displays, e.g., Finger-count Menus [2] or Bimanual Marking Menus [11]. Most of these interfaces group related tools in form of nested windows that float above the document. This helps users to remember where a particular tool can be found, but also requires them to spend considerable time and effort on organizing and arranging UI-components.

Challenge 2: Document Navigation. By looking through the eye of a spatially constrained display, the challenge of viewing large graphics documents at various levels of detail should not be underestimated. Users often have to switch between different views that can either depict close-ups (to inspect and edit details) or overviews (to maintain "overview" of the entire document). Such views are usually shown on the same display simultaneously, e.g., in form of an overview inset that occludes small parts of a detail view. Navigation (zoom \& pan) becomes significantly important in this scenario. It is usually accomplished directly within a view, e.g., by dragging the document with the mouse (pan) or by zooming in/out with keyboard shortcuts. With touch-enabled displays, a more direct way of interaction can be achieved. Yet, all interaction remains constrained to a $2 \mathrm{D}$ surface.

\subsection{Our Goal: Bridging Some of the Gaps}

In summary, the digital world offers a rich set of functionality with efficient ways to work with digital content. As a downside, it is restricted to a $2 \mathrm{D}$ workspace that provides only limited tangible affordances and thus does not leverage spatial arrangement 
as we are used to in the analog world. Aiming at closing this gap, we will next introduce the TUIP concept that combines some of the beneficial features of both worlds.

\section{The TUIP Concept}

The concept of Tangible User Interface Palettes (TUIP) is based on the traditional painter's metaphor, where a painter uses real-world tools like brushes and color palettes that are physically separated from the painting. With TUIP, we apply this idea to a digital tabletop. The tabletop screen shows graphics documents that users can edit by using digital pen input (see Figure 1). While tools (e.g., in form of menus) and the graphics document usually share the same screen space, we propose to decouple this space by making digital tool palettes physically tangible. We achieve this by using spatially aware handheld displays (Tangible UI Palettes) in different sizes and shapes that serve as physical representations for the otherwise virtual palettes. Users can take them into their hands and move and arrange them freely in 3D space and work with them using digital pen input. Thus, they bring back some of the advantages of a 3D work environment known from the analog world.

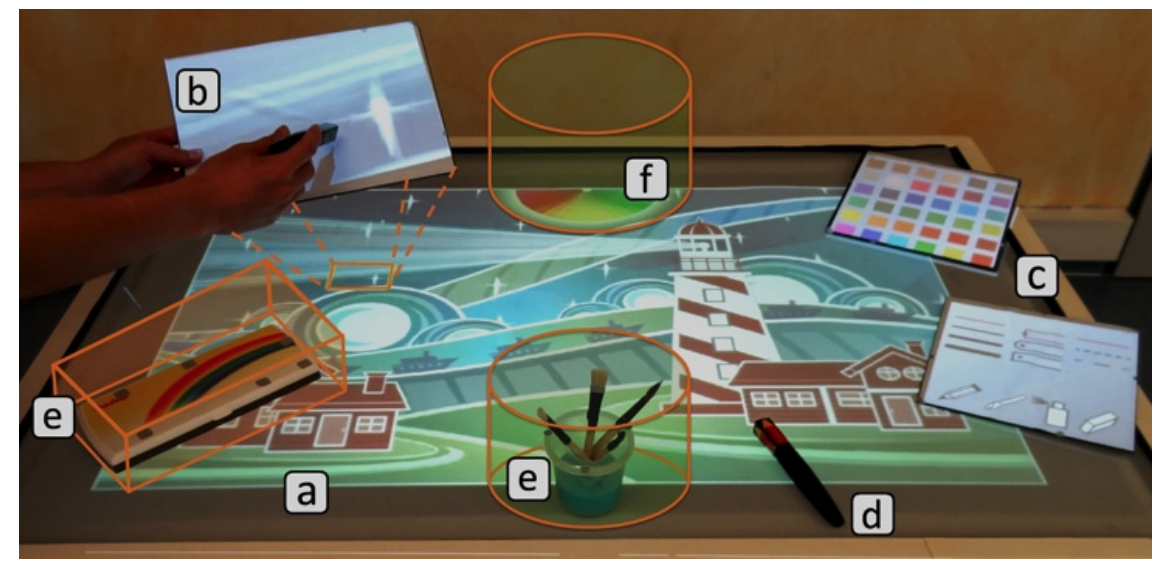

Fig. 1. Principle TUIP components: a tabletop with a graphics document (a), a handheld display with a detail view of that document (b), and handheld displays with tool menus (c). All displays are digital pen-enabled (d). Spatial work zones (e, f) are used as physical containers that can hold tools or document. They are represented by tangible proxies (e) or on-screen (f).

As all displays are dynamically updated depending on their position and orientation, instant visual feedback is displayed on them to reflect the state of tool usage. We take this concept further by introducing spatially bounded 3D interaction spaces (Work Zones) that exist above or beside the table. We use them as physical containers to group related functionality and documents. These work zones are visually represented either by tangible (physical) objects (see Figure 1e) or on-screen on the tabletop (see Figure 1f). Another important TUIP feature is digital pen input on all 
handheld displays and the tabletop, e.g., to simulate physical brush usage as known from the analog world. While in our work we only address workspaces with horizontal digital tables (standing and sitting usage), many of our concepts are general enough to be of use for other setups, e.g., tilted tabletops or wall-displays, as long as a horizontal surface for depositing handheld displays, pens and other physical objects is provided. In the following, we will discuss the TUIP design space and the concept of spatial Work Zones in more detail.

\subsection{Design Space}

The TUIP design space is illustrated in Figure 1. The main components are a single tabletop (see Figure 1a) and multiple handheld paper-like projection screens (see Figure $1 \mathrm{~b}, \mathrm{c})$ that are spatially tracked. These displays not only provide global and local views into a $2 \mathrm{D}$ information space, such as a graphics document (see Figure 1a,b), but can also switch dynamically to show functionality, e.g., in form of GUI palettes (see Figure 1c). For interacting with this system, we consider and support two input modalities: pen input and spatial input. Pen input is performed directly on the surface of tabletop and handheld displays with two degrees of freedom (2DOF). Technically, it is based on digital pen technology (Anoto), e.g., as it was proposed and used by Haller et al. for the Shared Design Space [13]. In our system, all displays are equipped with this technology (Figure 1d). Spatial input refers to the interaction with handheld displays by moving and rotating them through the physical space above or beside the table. With six degrees of freedom (6DOF), a rich set of interaction techniques becomes available, e.g., as categorized in [30]. One example for this is moving a display up and down for zooming. In order to make spatial interaction more explicit, we incorporated pressure-sensitive on-palette buttons, which further extends the interaction vocabulary. We also provide 6DOF spatial tracking for tangible proxies (used to represent spatial Work Zones).

One major advantage of such design space is the support of different input modalities that are close to what users are familiar with from everyday life. In particular, this includes drawing with (digital) pens and the spatial arrangement and organization of painting media and drawing tools (handheld displays). Clearly, both input strategies address a particular interaction goal better than the other one could possibly do. In this way, we are able to get a step closer to the overall goal of making the interaction more natural. The use of orthogonal input strategies also decreases the need of mode switches that are often perceived as being distractive. This helps take away mental load from users, thus potentially setting free intellectual capacity for more productivity and creativity. The combination of tabletop and multiple handheld displays enables the simultaneous use of shared and personal views (for both document and tools) and thus facilitates parallel work and co-located collaboration.

\subsection{Spatial Work Zones}

We propose to extend the interaction with spatially aware tangible displays by using proxemic interaction principles. We do this by introducing the concept of spatial 
Work Zones that are distinct spatial 3D regions above and around the table with individual spatial positions and extensions. They define an independent interaction space that can be explored by moving handheld displays into and through them. This either temporarily or permanently changes what is displayed on a handheld display (see Figure 2). In this way, spatial work zones serve as physical containers that we use for pooling related functionality or digital content.

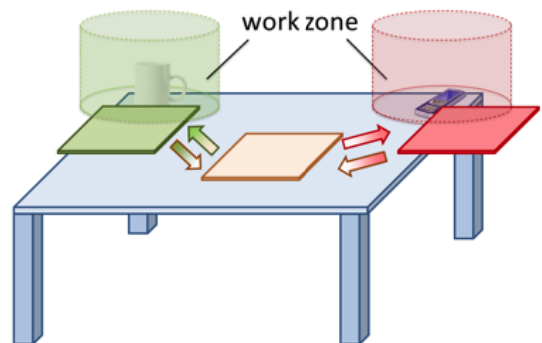

(a) Temporal Assignment

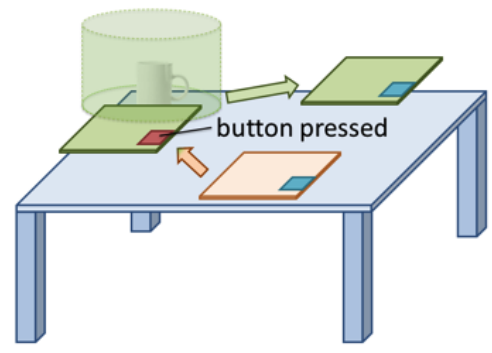

(b) Permanent Assignment

Fig. 2. Spatial Work Zones are spatially bounded regions above and beside the tabletop. They provide fast access to tool palettes and documents. Moving a handheld display into them instantly changes what is shown on the display. Leaving the Work Zone immediately restores the previous content (a). Pressing a special on-display button makes the change permanent (b). This allows for taking along a particular tool palette when leaving the Work Zone.

The spatial extension of a work zone is defined by its physical shape and size within the working environment. For simplicity, we usually use spheres with a fixed diameter of about $30 \mathrm{~cm}$. Each Work Zone has a center or spatial position that defines its location within the physical world. Users can freely manipulate this position and thus can arrange spatial Work Zones in their working environment as and when required. For this purpose, we introduce the concept of proxy representations. We support two types: tangible proxies and on-screen proxies that provide different levels of affordance. Users can seamlessly switch between these representations and use them, for example, to get an overview of available work zones or to move them to a new location. In the following, we will discuss their properties in more detail.

Tangible Proxies. Work Zones can be represented by real physical objects, which are optically tracked so that their spatial position is known to the system. These can be dedicated objects (e.g., a pot with brushes, see Figure 1e) only built and used for a specific purpose. They can also be generic objects like coffee mugs and post-its or other readily available objects. Tangible proxies offer a number of advantages. They are readily visible and thus can provide clues to the associated interaction. They are also freely moveable by physical means and allow for rearranging the associated Working Zone. This in particular includes the space beside the tabletop display, e.g., the table frame, where tangible proxies occlude no valuable screen space. Beyond that, personal objects like a wallet or mobile phone can be used to represent personalized Work Zones that provide, for example, access to confidential documents of a 
user or a customized tool configuration. One disadvantage of tangible proxies is that the system cannot actively move them, for example, to assist users by automatically restoring a previously defined Work Zone configuration. A current (technical) limitation is that visible markers need to be attached to each proxy object, though this could be solved in future iterations by relying on natural feature tracking techniques.

On-Screen Proxies. On-screen proxies are represented by regions situated on the tabletop display (see Figure 1f). Their advantage is that the system can change their location and appearance. This is useful, for example, when users want to restore their personal environment with a predefined Work Zone setup during the initialization phase or when the system needs to provide instant visual feedback about current state changes of the digital content associated with a Work Zone, e.g., the recently changed files of a Dropbox folder shared by multiple users. Users can freely arrange on-screen proxies on the tabletop display by performing pen- or finger-based drag gestures. As a downside, on-screen proxies usually occlude the document visible on the tabletop display. This problem can be lowered with well-established methods like partial transparency, context-sensitive fade-ins or showing them only when a handheld display moves towards them. Another problem is that spatial Work Zones represented by on-screen proxies are restricted to the direct proximity of the screen, thus limiting the user's freedom of arranging Work Zones farther away from the table. A possible approach to soften this problem is to use direction signs as a visual representation, e.g., an arrow could indicate that there is a Work Zone on the right side of the table.

Switching between Representations. A seamless switch between both types of representations can be achieved by performing a double-tap gesture with a tangible proxy on the tabletop screen. This means that the user must slightly knock on the tabletop surface with a physical proxy object in her hands. This works in both directions:

- Screen-to-Tangible: When the double-tap occurs directly on an on-screen proxy, its associated Work Zone will be transferred to the tangible proxy and the onscreen proxy is removed from the screen.

- Tangible-to-Screen: After a double-tap gesture is performed somewhere on the tabletop surface, an on-screen proxy is created at this position and the tangible proxy's Work Zone is transferred to this new on-screen proxy. This also implies that the tangible proxy is no longer linked to the Work Zone.

\section{$5 \quad$ Use Cases}

We continue by presenting several use cases that demonstrate how TUIP principles can support tool organization and document navigation using the example of graphics applications. Since TUIP combine techniques that have been presented previously, in particular Paper Windows [15] and PaperLens [29], we will first show how these fit into the overall concept (Use Case $1 \& 2$ ). We will then present what we consider our major contribution: the use of spatial Work Zones as a more transient way of working with tools and document views using handheld displays (Use Case $3 \& 4$ ). 


\subsection{Use Case 1: Document Navigation}

To support the work with large graphics documents, efficient document navigation techniques are needed that allow users to efficiently zoom and pan (Challenge 2). We address this by physically detaching overview views from detail views and distribute them to the tabletop and several handheld displays (see Figure 3), similar as it was demonstrated by us in PaperLens [29]. In contrast to previous work, with TUIP we support both navigation (by moving a display) and drawing (by digital pen input). We therefore make use of explicit activation of spatial interaction, in our case, by pushing an on-display button. This guarantees that navigation only occurs when users really intend to, which is an important requirement for graphics document editing.

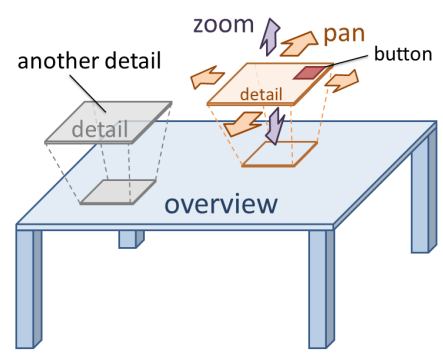

(a) Spatial input-based navigation (concept)

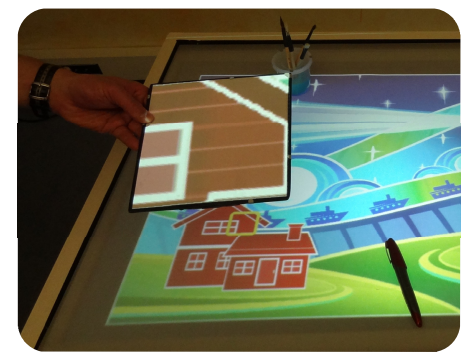

(b) Handheld zoomed-in view of a document

Fig. 3. Document navigation relies on spatial positions of handheld displays (up/down: zoom, horizontal: pan). This is similar to a concept proposed by us in PaperLens [29], except that we make the technique explicit (by pushing/holding an on-display button).

\subsection{Use Case 2: Inter-display Transfer of Palettes}

A major design goal of TUIP is to physically decouple menus from screen space in order to take them into the hands or to lay them out on the desk by still maintaining complete interactivity. This particularly addresses Challenge 1 (Tool Management). In this context, the seamless transfer of digital content between tabletop and handheld displays (in all thinkable combinations) is a crucial requirement. In Paper Windows [15], Holman et al. suggested to achieve this by placing a handheld display (in their
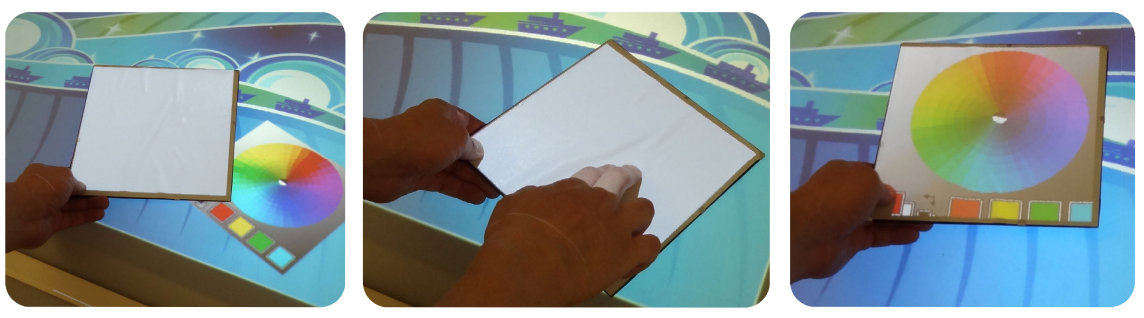

Fig. 4. As proposed by [15], the transfer of virtual windows from one screen, e.g., the tabletop (left), to another one (e.g., a handheld display) is done via a rubbing gesture (middle). This allows users to pick up digital tools (right) or parts of the document in a tangible manner. 
case a digital piece of paper) directly on top of a virtual window and then to trigger the transfer by a rubbing gesture. We applied this concept to TUIP (see Figure 4).

\subsection{Use Case 3: Quick Access to Tools by Using Work Zones}

In the WIMP world, shortcuts such as keystroke combinations and mouse gestures provide quick access to specific interactions or frequently used tools. Inspired by the analog world, where artists collect their favorite pens and brushes in a coffee mug and put it right next to them on the desk for easy access, we propose to use spatial Work Zones (see Figure 2) for grouping related functionality (e.g., different color palettes for color management). Users can freely arrange these Work Zones on the table via tangible proxies, e.g., a jar with brushes (see Figure 1e).

A spatial Work Zone can contain multiple tool palettes simultaneously. We propose to organize them as a multi-layer stack (see Section 2.5). Moving a handheld display into a Work Zone triggers the multi-layer stack mechanism, i.e., no button is needed for any mode switch (see Figure 5a). The only temporarily visible tool palettes are instantly functional, e.g., ready to be used by a digital pen (see Figure 5b). As soon as the display is moved out of the Work Zone, the original content of the handheld display will be restored. This allows for making a series of adjustments in a streamlined manner, which can also involve more than one Work Zone, see Figure 5a.

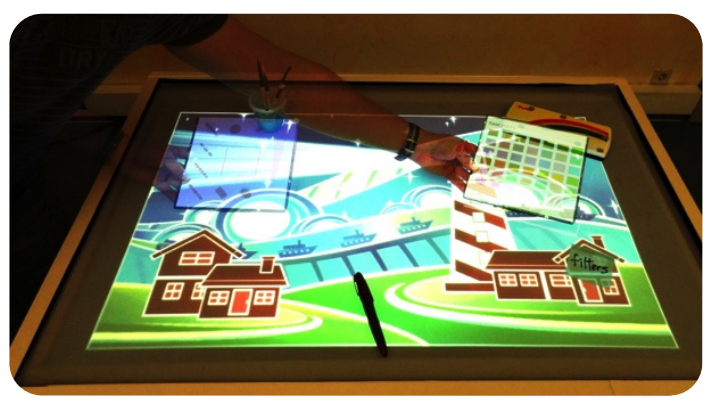

(a) Switching between tool palettes by moving a handheld display from one Work Zone (left) into another one (right).

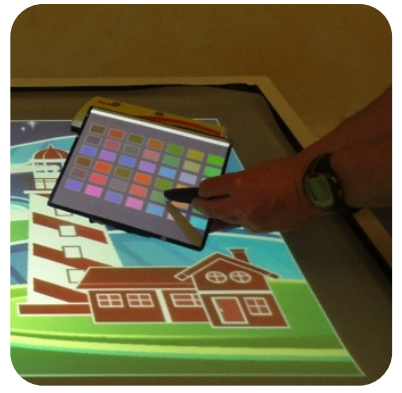

(b) Picking a color from a tool palette with a digital pen.

Fig. 5. By entering a spatial Work Zone with a handheld display the user gains quick access to available tool palettes (a). Any palette visible on a display is instantly interactive (b).

Permanent assignments (see Figure 2b) are achieved by double-clicking an ondisplay button when a desired GUI palette is visible. This allows users to put down a display on the table for other tasks, yet able to access the tool palette without going through the whole process again. A button press (and hold) prior to moving into a Work Zone achieves a transfer into the opposite direction. This lets users customize Work Zones by spatially "dragging-and-dropping" a GUI palette into the Work Space's multi-layer stack.

As proposed in [29,31], we visually guide users during the exploration of a multilayer stack by fading in a height indicator on the right side of the display that shows 
all layers associated with a Work Zone (see Figure 6a). A red cursor bar in the height indicator shows the approximate position of the display within the stack. The height indicator automatically fades out when the user is not moving the display up/down for a certain time ( 2 seconds). We achieve a smoother user experience by blending adjacent tool palettes when a display reaches another layer of the stack (see Figure 6b).

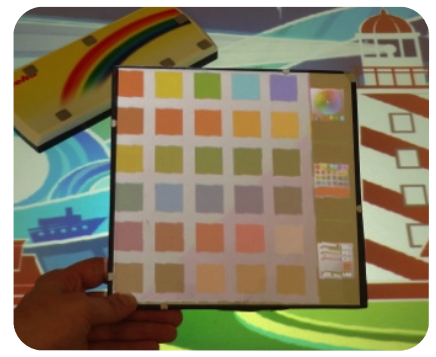

(a) Height indicator (right)

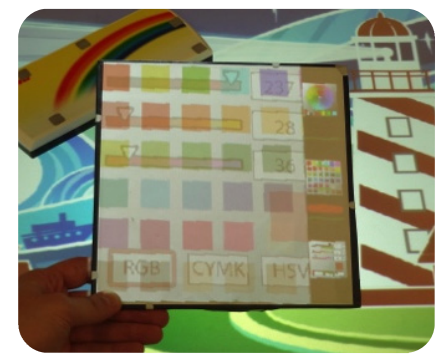

(b) Blending of adjacent layers

Fig. 6. A height indicator on the display provides visual orientation during the exploration of a multi-layer stack (a). The gradual visual blending of two adjacent palettes (when a display is about to cross a layer border) helps users to stay within a particular layer more easily (b).

\subsection{Use Case 4: Quick Access to Graphics Layers by Using Work Zones}

Spatial Work Zones can also provide quick access to other digital items commonly found in graphics editors, including parts of the graphics document. One example for this are graphics layers that store independent graphics components separately, thus allowing for a more flexible management of the document. The multi-layer stack matches this concept perfectly, e.g., by filling a dedicated Work Zone with all graphics layers of an image document. Similar to the previous use case, the exploration of individual layers is accomplished by entering the Work Zone with a display. Moving it up and down switches between layers. Here, different visibility configurations are possible, e.g., current layer only or all layers below the current one. When held vertically, the display provides fast access to an overview of all layers of a document, e.g., similar to Adobe Photoshop's Layer Panel. There is one problem, though: the work with vertically held handheld displays is not comfortable for prolonged usage. Fortunately, this can be tackled by making the overview permanent (e.g., by pressing an on-display button). This allows users to hold the display more conveniently, while they are working with it, e.g., to reorder or hide particular layers using pen or finger input.

By assigning a special tangible proxy to the "graphics layers" Work Zone, users can freely arrange the Work Zone to suit a particular task, e.g., by putting the proxy down somewhere on the table. This provides access to different image regions in a global vs. local scope. For example, when the Work Zone is positioned on top of a particular detail of the image (e.g., the left eye of a face), the Work Zone only provides access to layers involving that eye and its surroundings. In this way, users have quick access to relevant layers of an image detail. In contrast, when the "graphics 
layers" Work Zone is moved besides the tabletop display, the focus changes to global features, i.e., now scaled down miniature versions of layers are shown on the handheld display. This provides fast access to global layer management functionality, e.g., useful for organizing which layers are actually visible on the tabletop.

\section{First Prototype}

We have implemented a prototypic TUIP-system that relies on projective (paper-like) display technology, see Section 2.2. We use an infrared (IR)-based tracking approach, where all tracked devices (handheld displays and tangible proxies) have IR-reflective markers attached in distinguishable configurations. The system also includes a gesture recognizer to detect distinct move patterns (e.g., flipping gestures) of tracked devices. A ceiling-mounted projector is responsible for projecting digital image content onto handheld displays. Due to a skewed projection frustum, it also allows for projecting image content onto (almost) vertically positioned displays. A second projector projects from underneath the tabletop. An Anoto pattern is attached to both the tabletop and the handheld displays to support digital pens. Some of the handheld displays were equipped with a pressure-sensitive button (Arduino Xbee) that can wirelessly communicate state changes to the system. We use theses buttons primarily for explicit activation of spatial input-based navigation (see Figure 3a) and permanent assignment of tool palettes (see Figure 2b).

Considering the constraints of a research prototype, we did not spend much effort in implementing a real graphics editor application. Our focus was rather on illustrating the basic concepts of a TUIP system. This includes spatial Work Zones, spatial input-based navigation with handheld displays, and some basic tool palettes, e.g., for color and brush selection. Aside from this, pixel resolutions on handheld displays are rather limited due to the projector-based approach (about 65 pixels/inch). Thus, precision is by far not sufficient enough for serious work, e.g., as compared to modern tablets. However, the support of specific form factors of hand displays was more important to us. This includes small display weights (similar to cardboard), different display shapes and sizes, the avoidance of display bezels, the support of screens on the backside of displays, and the seamless support of pen-based drawing on all involved displays. Despite the technical limitations, we are confident that our prototype is adequate for basic testing of the underlying concepts. We expect that many of these limitations can be solved in the future, e.g., by using high-resolution projectors or organic light-emitting diode (OLED) display technology.

\section{Initial User Experience}

We have collected early user feedback on our prototype from four regular users of desktop graphics editors like Photoshop and Illustrator. All of them are experienced with touch- and pen-based interfaces. During an initial introduction to the prototype and its capabilities, we asked the participants not to get too much distracted by the rather low image quality or the limited functionality of the system. Rather, we asked 
them to assess the overall concept and whether they could image using similar techniques for regular work. All four users worked freely with the system for about 20 minutes. They performed a series of tasks requiring them to navigate within a graphics document and to switch between different tools frequently using TUIP techniques. After observing them interacting with the prototype, an interview was conducted to further elicit their experience and expectations for future use of the proposed system.

\subsection{General Impression}

Participants praised the seamless integration of pen-input on all displays as well as the support of spatial arrangement of digital content by using multiple handheld displays. Negative feedback was almost entirely related to the technical limitations of the prototype, but not to the underlying concept, which in general was assessed very positively. Although participants felt comfortable using the system for sketching and scribbling, they were (at this stage of implementation) reluctant in using it for more serious work, because (as they stated) this usually requires a much higher level of accuracy and a broader set of implemented tools, which was not yet supported by our prototype. However, users also said that they could imagine using a similar system for daily work once these problems are addressed properly. One user suggested that the system might be more preferable if the tabletop display could be tilted. Later, the same user acknowledged that a similar effect could be achieved by holding a handheld display accordingly and that maintaining a horizontal table surface provides adequate room for depositing pens, tangible proxies and handheld displays.

\subsection{Document Navigation with Handheld Displays}

Users particularly liked the possibility of decoupling specific regions of the image document from the main tabletop by transferring them to one or more handheld displays (see Figure 3). This allowed them to take a particular image detail into their hands, e.g., the left eye of a woman's face, while the context (e.g., the woman's face) was still visible on the tabletop. All participants stated that this considerably helped them to maintain focus and overview. Users also appreciated that image details on handheld displays were directly editable by pens, allowing them to draw in a zoomedin view, while the overall image on the tabletop was updated live. Participants found it easy and even natural to select a specific image detail on a handheld display by moving the display through the air above the table (accompanied by pushing an ondisplay button to activate spatial input). After some minutes of practice, all users developed a fairly good understanding about the mapping of zoom level and height above the tablet surface. This effectively allowed them to directly jump to a specific zoom level by just holding the display at a particular height. Three users suggested that on-display buttons should provide tactile feedback to better reflect whether a button (and thus spatial input) was activated or not. Two users asked for additional support of touch-based navigation that they thought would be more appropriate for slightly re-centering a picture detail on a display when drawing with a pen. 


\subsection{Tool Organization with Spatial Work Zones}

Participants described the concept of spatial Work Zones as intuitive and very useful for organizing digital content. In particular, they praised the ability to quickly switch between views of the document and tool palettes by simply entering/leaving one of the Work Zones. They also liked the possibility to freely arrange Work Zones on the table surface by using physical objects, including putting them away if not needed. One user suggested using Work Zones for representing special folders, e.g., as containers for "good" and "bad" photos in a photo collection. Users generally found it easy to pick one of the four tool palettes that we had attached to each Work Zone (organized as a multi-layer stack). However, users wished for more customization options regarding the number of palettes and their order within the palette-stack (something that we had not focused on in our prototype, yet). When working with the prototype, we observed that participants employed two distinct work patterns. The first pattern was employed when users were working on a single handheld display showing a detailed view of the document. Here, users approached spatial Work Zones repeatedly for quick (temporary) tools access (e.g., changing colors and pen thicknesses) by using temporary assignment (see Figure 2a). Participants stated that they particularly liked that the document view was instantly restored on the handheld display whenever they left a Work Zone. In the second pattern, users assigned two or three tool palettes to several handheld displays by using permanent assignment (see Figure $2 \mathrm{~b}$ ) prior to the actual drawing task. Then, they put the handheld displays on the table for convenient access. With that they used a digital pen to choose tools from handheld displays and to draw on the tabletop.

\section{Conclusion and Future Work}

In this work, we brought spatial affordances back to the digital world of graphics editing in a tabletop environment. Previous approaches have partially addressed this issue by augmenting digital tables with static (printed) physical palettes, for example, based on digital pen and paper technology. By using principles of spatial interaction and tangible affordances, we extended this idea by contributing dynamic (projected) Tangible UI Palettes and ways to access them. In particular, we proposed the concept of spatial Work Zones that are represented by tangible proxies or on-screen on the tabletop. We presented different use cases that illustrate the usefulness of our techniques. These use cases specifically addressed secondary interaction goals in graphics editing, like providing quick access to tool palettes and graphics layers, but can also be applied beyond this application domain. We implemented a prototype demonstrating our concepts. Early user feedback on the prototype was promising, especially concerning the ability to have fast access to digital content in a transient manner and the support for switching between tool menus and document views seamlessly on the very same handheld display.

In the future, we plan to drastically increase the set of available tools and enhance the visual quality and accuracy of the prototype with the goal of conducting a formal user study to further evaluate the efficacy of our techniques, including better support 
for inter-display interaction and more sophisticated techniques for spatial Work Zones. One particular advantage of the TUIP concept is that it is easily extendable and integrates well with multi-touch input, e.g., by using tablets. While in this work we primarily focused on spatial and pen input, we plan on integrating finger-based input, e.g., for precise zooming and panning of details on handheld displays.

Acknowledgements. This work was funded by the German Ministry of Education and Science (BMBF) project ViERforES-II (01IM1000 2B). We thank Hannes Witt for his help in implementing and evaluating the prototype.

\section{References}

1. Anoto Group AB - Website, http: / / www . anoto.com

2. Bailly, G., Lecolinet, E., Guiard, Y.: Finger-count \& radial-stroke shortcuts: 2 techniques for augmenting linear menus on multi-touch surfaces. In: Proc. CHI 2010, pp. 591-594. ACM Press (2010)

3. Ballendat, T., Marquardt, N., Greenberg, S.: Proxemic interaction: designing for a proximity and orientation-aware environment. In: Proc. ITS, pp. 121-130. ACM Press (2010)

4. Bencina, R., Kaltenbrunner, M.: The design and evolution of fiducials for the reacTIVision system. In: Proc. Generative Systems in the Electronic Arts 2005 (2005)

5. Bier, E.A., Stone, M.C., Pier, K., Buxton, W., DeRose, T.D.: Toolglass and magic lenses: the see-through interface. In: Proc. SIGGRAPH 1993, pp. 73-80. ACM Press (1993)

6. Block, F., Haller, M., Gellersen, H., Gutwin, C., Billinghurst, M.: VoodooSketch: extending interactive surfaces with adaptable interface palettes. In: Proc. TCI 2008, pp. 55-58. ACM Press (2008)

7. Brandl, P., Leitner, J., Seifried, T., Haller, M., Doray, B., To, P.: Occlusion-aware menu design for digital tabletops. Ext. Abstracts CHI 2009, pp. 3223-3228. ACM Press (2009)

8. Dietz, P., Leigh, D.: DiamondTouch: a multi-user touch technology. In: Proc. UIST 2001, pp. 219-226. ACM Press (2001)

9. Elrod, S., Bruce, R., Gold, R., Goldberg, R., Halasz, F., Janssen, W., Lee, D., McCall, K., Pedersen, E., Pier, K., Tang, J., Welch, B.: Liveboard: a large interactive display supporting group meetings, presentations, and remote collaboration. In: Proc. CHI 1992, pp. 599607. ACM Press (1992)

10. Greenberg, S., Fitchett, C.: Phidgets: easy development of physical interfaces through physical widgets. In: Proc. UIST 2001, pp. 209-218. ACM Press (2001)

11. Guimbretière, F., Nguyen, C.: Bimanual marking menu for near surface interactions. In: Proc. CHI 2012, pp. 825-828. ACM Press (2012)

12. Hall, E.T.: The Hidden Dimension. Anchor Books (1966)

13. Haller, M., Brandl, P., Leithinger, D., Leitner, J., Seifried, T., Billinghurst, M.: Shared design space: Sketching ideas using digital pens and a large augmented tabletop setup. In: Pan, Z., Cheok, D.A.D., Haller, M., Lau, R., Saito, H., Liang, R. (eds.) ICAT 2006. LNCS, vol. 4282, pp. 185-196. Springer, Heidelberg (2006)

14. Harrison, C., Dey, A.K.: Lean and zoom: proximity-aware user interface and document magnification. In: Proc. CHI 2008, pp. 507-510. ACM Press (2008)

15. Holman, D., Vertegaal, R., Altosaar, M., Troje, N., Johns, D.: Paper windows: interaction techniques for digital paper. In: Proc. CHI 2005, pp. 591-599. ACM Press (2005) 
16. Holmquist, L.E., Mattern, F., Schiele, B., Alahuhta, P., Beigl, M., Gellersen, H.-W.: Smart-its friends: A technique for users to easily establish connections between smart artefacts. In: Abowd, G.D., Brumitt, B., Shafer, S. (eds.) UbiComp 2001. LNCS, vol. 2201, pp. 116-122. Springer, Heidelberg (2001)

17. Ishii, H., Ullmer, B.: Tangible bits: towards seamless interfaces between people, bits and atoms. In: Proc. CHI 1997, pp. 234-241. ACM Press (1997)

18. Izadi, S., Hodges, S., Taylor, S., Rosenfeld, D., Villar, N., Butler, A., Westhues, J.: Going beyond the display: a surface technology with an electronically switchable diffuser. In: Proc. UIST 2008, pp. 269-278. ACM Press (2008)

19. Johanson, B., Fox, A., Winograd, T.: The Interactive Workspaces Project: Experiences with Ubiquitous Computing Rooms. IEEE Pervasive Computing 1(2), 67-74 (2002)

20. Kakehi, Y., Naemura, T.: UlteriorScape: Interactive optical superimposition on a viewdependent tabletop display. In: Proc. TABLETOP 2008, pp. 189-192 (2008)

21. Kirsh, D.: The intelligent use of space. Artificial Intelligence - Special Volume on Computational Research on Interaction and Agency, Part 2 73(1-2), 31-68 (1995)

22. Kray, C., Rohs, M., Hook, J., Kratz, S.: Group coordination and negotiation through spatial proximity regions around mobile devices on augmented tabletops. In: Proc. TABLETOP 2008, pp. 1-8 (2008)

23. Kurtenbach, G., Buxton, W.: Issues in combining marking and direct manipulation techniques. In: Proc. UIST 1991, pp. 137-144. ACM (1991)

24. Lee, J.C., Hudson, S.E., Summet, J.W., Dietz, P.H.: Moveable interactive projected displays using projector based tracking. In: Proc. UIST 2005, pp. 63-72. ACM Press (2005)

25. Leitner, J., Powell, J., Brandl, P., Seifried, T., Haller, M., Dorray, B., To, P.: Flux: a tilting multi-touch and pen based surface. Ext. Abstracts CHI 2009, pp. 3211-3216. ACM Press (2009)

26. Morris, M.R., Ryall, K., Shen, C., Forlines, C., Vernier, F.: Beyond "social protocols": multi-user coordination policies for co-located groupware. In: Proc. CSCW 2004, pp. 262265. ACM Press (2004)

27. Ryokai, K., Marti, S., Ishii, H.: I/O Brush: Drawing with Everyday Objects as Ink. In: Proc. CHI 2004. ACM Press (2004)

28. Song, H., Benko, H., Guimbretiere, F., Izadi, S., Cao, X., Hinckley, K.: Grips and Gestures on a Multi-Touch Pen. In: Proc. CHI, pp. 1323-1332. ACM Press (2011)

29. Spindler, M., Stellmach, S., Dachselt, R.: PaperLens: advanced magic lens interaction above the tabletop. In: Proc. ITS 2009, pp. 69-76. ACM Press (2009)

30. Spindler, M., Tominski, C., Schumann, H., Dachselt, R.: Tangible views for information visualization. In: Proc. ITS 2010, pp. 157-166. ACM Press (2010)

31. Spindler, M., Martsch, M., Dachselt, R.: Going Beyond the Surface: Studying Multi-Layer Interaction Above the Tabletop. In: Proc. CHI 2012. ACM Press (2012)

32. Sellen, A.J., Harper, R.H.R.: The Myth of the Paperless Office. The MIT Press (2001)

33. Ullmer, B., Ishii, H.: The metaDESK: Models and Prototypes for Tangible User Interfaces. In: Proc. UIST. ACM Press (1997)

34. Vandoren, P., Van Laerhoven, T., Claesen, L., Taelman, J., Raymaekers, C., Van Reeth, F.: IntuPaint: Bridging the gap between physical and digital painting. In: Proc. TABLETOP 2008, pp. 65-72 (2008)

35. Weiser, M.: The computer for the 21st century. Scientific American 265(3), 66-75 (1991)

36. Wilson, A., Benko, H.: Combining Multiple Depth Cameras and Projectors for Interactions On, Above, and Between Surfaces. In: Proc. UIST 2010, pp. 273-282. ACM Press (2010) 